\title{
¿Son los adultos un modelo de conducta influyente en los hábitos de actividad física de los menores? Un estudio observacional de la población española Are adults an influential role model in children's physical activity habits? An observational study of the Spanish population \\ Brais Boente Antela, Raquel Leirós-Rodríguez, Jose Luis García-Soidán \\ Universidad de Vigo (España)
}

\begin{abstract}
Resumen: Este estudio se llevó a cabo con los objetivos de determinar la prevalencia del sobrepeso y la obesidad de los menores españoles; identificar las posibles relaciones existentes entre éstas y los hábitos de AF en los adultos; y explicar si dichas relaciones tienen como origen variables socio-económicas para la muestra objeto de estudio. Para alcanzarlos se realizó un estudio observacional transversal basado en los datos extraídos de la última Encuesta Nacional de Salud de España disponible (2017) de 7598 encuestas respondidas por un adulto que viviese en un domicilio en el que se registrase la existencia de un menor mayor de 5 años. La proporción de menores con sobrepeso desciende a medida que el nivel de estudios del adulto es más alto. Asimismo, la cantidad de menores con infrapeso incrementa ligeramente con el aumento de nivel de estudios del adulto. En general, los menores realizan con más frecuencia actividad física que los adultos (38.1\% de adultos sedentarios frente a $13.7 \%$ de menores) y dicha frecuencia aumenta a medida que el nivel de estudios del adulto es más elevado. Por tanto, los resultados obtenidos muestran que los menores españoles tienden a replicar el estilo de vida (lo que incluye los hábitos de actividad física) de los adultos con los que conviven. Lo que convierte a los adultos en la población diana sobre la que incidir políticas sanitarias de prevención primaria y secundaria para mejorar su salud y la de los niños que los rodean.
\end{abstract}

Palabras clave: Actividad motora; Conducta sedentaria; Estilo de vida; Pediatría; Salud Pública.

\begin{abstract}
This study was carried out with the objectives of determining the prevalence of overweight and obesity in Spanish minors; identify the possible relationships between them and PA habits in adults; and explain whether these relationships have socio-economic variables for the sample under study. To achieve them, a cross-sectional observational study was carried out based on data from the last available Spanish National Health Survey (2017) of 7598 surveys answered by an adult living in a home in which the existence of a minor older than 5 years. The proportion of overweight minors decreases as the level of education of the adult is higher. Likewise, the number of underweight children increases slightly with the increase in the level of education of the adult. In general, minors engage in physical activity more frequently than adults (38.1\% of sedentary adults compared to $13.7 \%$ of minors) and this frequency increases as the level of education of the adult is higher. Therefore, the results obtained show that Spanish minors tend to replicate the lifestyles related to the physical activity of the adults with whom they live. This makes adults the target population to influence primary and secondary prevention health policies to improve their health and that of the children around them.
\end{abstract}

Key words: Motor activity; Life style; Pediatrics; Public Health; Sedentary behavior.

\section{Introducción}

El sobrepeso y la obesidad están asociados con consecuencias adversas para la salud (diabetes tipo 2, enfermedades cardiovasculares y un mayor riesgo de cáncer, entre muchos otros) y efectos económicos negativos para el individuo que los padece y para la sociedad a la que éste permanece (Cawley, 2015). Las tendencias estimadas en Europa, por ejemplo, indican que la prevalencia promedio de sobrepeso (y obesidad) ha alcanzado el 54\% (24\%) en mujeres y alrededor del 66\% (23\%) en hombres (NCD Risk Factor Collaboration, 2017). Paralelamente, se estima que en 2020 la prevalencia del sobrepeso infantil alcance el 10\% y el 20\% para la población de menores pre-escolares y escolares, respectivamente (De Onis, Blössner, \& Borghi, 2010; Wang \& Lim, 2012). Al mismo tiempo, se ha demostrado que la obesidad infantil se correlaciona con su permanencia en la etapa adulta y con mortalidad prematura (Reilly \& Kelly, 2011).

La práctica de $\mathrm{AF}$ conlleva una serie de reacciones fisiológicas entre las que se encuentra la segregación de neurotransmisores y hormonas que facilitan el desarrollo intelectual al facilitar la generación de nuevas neuronas y multiplicando y fortaleciendo las conexiones neuronales ya existentes entre las áreas encefálicas relacionadas con la

Fecha recepción: 24-03-17. Fecha de aceptación: 29-06-20

Jose Luis García Soidan

jlsoidan@uvigo.es memoria y el aprendizaje (Erickson et al., 2011; Gearin \& Fien, 2016; Rosa, García, \& Martínez, 2020). Estos fenómenos, presentes a lo largo de toda la vida, cobran especial relevancia en los niños, en los que la práctica de AF provoca mejoras en aptitudes intelectuales básicas como el control emocional, la memoria o la capacidad de adaptación a diferentes tareas y entornos que son imprescindibles para el óptimo desarrollo académico y emocional (Gómez Pinilla \& Hillman, 2013; Hillman et al., 2014).

El origen del sobrepeso es multifactorial y tras él subyace un complejo sistema de factores interconectados (MartínezBaena, Mayorga-Vega, \& Viciana, 2018; Finegood, Merth, \& Rutter, 2010). En el caso de los niños, dichos factores incluyen el consumo y el gasto diario de energía, los patrones de actividad física (AF) y factores genéticos, ambientales, valores socio-culturales, clase social, factores económicos, estrés... (Blundell, Dulloo, Salvador, \& Fruhbeck, 2014).

En 2015, Lane et al. (2015) relacionaron, por primera vez de manera contrastada, el estilo de vida de los padres (antes y después de la concepción) como factor de gran impacto en el desarrollo de sobrepeso y obesidad de los niños. Paralelamente, se ha identificado que en Europa (y especialmente en los países del Sur como España), las familias con bajo nivel socio-económico y los niños con padres con sobrepeso $\mathrm{u}$ obesidad tienen mayor predisposición a desarrollar mayores niveles de Índice de Masa Corporal (IMC) (Godoy-Cumillaf, Bruneau-Chávez, Fuentes-Merino, \& Ojeda-Santana, 2020; Manios et al., 2018). 
Teniendo en cuenta todo lo anterior, el incremento de la práctica de AF en el sector poblacional infantil se ha vuelto una prioridad de Salud Pública en la última década y, aunque se han realizado investigaciones que confirman la relación entre la conducta parental en relación a la AF y su reflejo en la de los niños hay una carencia en la explicación de los factores que den explicación a dicho fenómeno y a la gran varianza en los resultados obtenidos en diferentes poblaciones (Adamo, Langlois, Brett, \& Colley, 2012; Kaseva et al., 2017; Schoeppe, Röbl, Liersch, Krauth, \& Walter, 2016). Por un lado, hay que tener en cuenta el cambio de paradigma en los modelos de paternidad: pasando de los más tradicionales (democrático, permisivo, negligente y autoritario) al más reciente de «estilo transformador» (Morton, Wilson, Perlmutter, \& Beauchamp, 2012). El estilo transformacional se caracteriza por la provisión de apoyo, aliento y afecto positivo hacia los niños, la transmisión de la idea de respetar a los demás, junto con el establecimiento de normas de conducta apropiadas que, si se rompen, no son contrarrestados por la coacción ni medidas disciplinarias (Morton, Wilson, Perlmutter, \& Beauchamp, 2012; Popper, \& Mayseless, 2003). Llevar a cabo un liderazgo transformador en la familia, se asocia con el mantenimiento de una dieta saludable y mayor práctica de AF en el tiempo libre y como actividad de ocio como método para aumentar la adherencia a estilos de vida saludables y el alcance de un mejor autoconcepto físico y mayores niveles de satisfacción vital (Álvarez, Castillo, Molina-García, \& Balagué, 2016; Verma, Eklund, Arthur, Howle, \& Gibson, 2019).

Por todo ello, se consideró necesaria la realización del presente estudio con los objetivos de (a) determinar la prevalencia del sobrepeso y la obesidad de los menores españoles; (b) identificar las posibles relaciones existentes entre éstas y los hábitos de AF en los adultos; y (c) explicar si dichas relaciones tienen como origen variables socio-económicas para la muestra objeto de estudio.

\section{Material y Método}

\section{Diseño experimental}

Este estudio observacional transversal se basó en los datos extraídos de la última Encuesta Nacional de Salud de España (ENS) disponible (2017) realizada por el Instituto Nacional de Estadística (INE) y el Ministerio de Sanidad, Consumo y Bienestar Social del Gobierno de España para recoger información sanitaria relativa a la población residente en España. Es una investigación de periodicidad quinquenal que permite conocer numerosos aspectos de la salud de los ciudadanos a nivel nacional y autonómico, y planificar y evaluar las actuaciones en materia sanitaria. La encuesta se realizó (mediante muestreo sistemático y con equiprobabilidad de ser seleccionados dentro de sus correspondientes secciones censales a 37500 viviendas familiares de todo el territorio nacional entre octubre de 2016 y octubre de 2017 (Instituto Nacional de Estadística, 2020).

\section{Muestra}

Finalmente, la ENS de 2017 en España incluyó a una población de 23860 personas. El proceso de muestreo se realizó en todas las provincias españolas. La encuesta utilizó un muestreo de tres etapas con estratificación. Las unidades de la primera etapa fueron secciones censales. Las unidades de la segunda etapa fueron las viviendas. Por último, se seleccionó a una persona adulta dentro de cada hogar. Los cuestionarios se completaron realizando entrevistas personales. Detalles sobre la metodología de diseño y aplicación de la ENS pueden consultarse en el sitio web del INE (Instituto Nacional de Estadística, 2020).

En este estudio se han empleado los datos procedentes de 7598 encuestas respondidas por un adulto que viviese en un domicilio en el que se registrase la existencia de un menor mayor de cinco años. Los menores de dicha edad fueron excluidos porque la cuantificación de la AF realizada por menores de esta edad sin monitorización objetiva ni observación directa carece de fiabilidad (Oliver, Schofield, \& Kolt, 2007), por lo que los menores estudiados en esta investigación tenían un rango de edad de entre cinco y 18 años. Por tanto, el único criterio de inclusión empleado fue que los adultos que hubieran completado la ENS de 2017 y que conviviesen con un menor de edad. Y, como criterio de exclusión, que el menor de edad que conviviese con el participante en la ENS fuera menor de cinco años. De la muestra total, incluyendo tanto a adultos como a menores, la distribución por sexos fue de $50.8 \%$ de hombres y $49.2 \%$ de mujeres y la edad media de $43.9 \pm 25.2$ años.

\section{Variables de estudio}

Como variables dependientes se consideraron las frecuencias de actividad física en el tiempo libre del adulto y del menor: estas variables en la ENS se plantean a través de la pregunta ¿Cuál de estas posibilidades describe mejor la frecuencia con la que realiza alguna actividad física en su tiempo libre? Teniendo como respuestas posibles: (a) no hace ejercicio; (b) hace alguna actividad física o deportiva ocasional; (c) varias veces al mes; o (d) varias veces a la semana (Instituto Nacional de Estadística, 2020).

Las variables independientes elegidas fueron: (a) sexo; (b) edad (años); (c) peso (kg); (d) nivel de estudios del adulto, categorizado en tres grupos como estudios primarios y/o Educación Secundaria Obligatoria, Bachillerato y/o Ciclos Formativos y estudios universitarios; (e) Índice de Masa Corporal (IMC) $\left(\mathrm{kg} / \mathrm{m}^{2}\right)$, derivado del cálculo del peso y altura que los participantes informaron en la entrevista y categorizado para los adultos como infrapeso (IMC $<18.5$ $\left.\mathrm{kg} / \mathrm{m}^{2}\right)$, normopeso $\left(18.5 \mathrm{~kg} / \mathrm{m}^{2}\right.$ e» $\left.\mathrm{IMC}<25 \mathrm{~kg} / \mathrm{m}^{2}\right)$; sobrepeso $\left(25 \mathrm{~kg} / \mathrm{m}^{2}\right.$ e» IMC $\left.<30 \mathrm{~kg} / \mathrm{m}^{2}\right)$ y obesidad (IMC e» $30 \mathrm{~kg} / \mathrm{m}^{2}$ ). Para la población de entre 5 y 14 años se siguió la clasificación propuesta por la World Obesity Federation (Cole, Flegal, Nicholls, \& Jackson, 2007) que categoriza como peso insuficiente que categoriza como peso insuficiente aquel valor de IMC que no alcanza el valor mínimo como saludable para su sexo y edad. Tanto la condición de sobrepeso como la de obesidad son determinadas a partir de +1 desviación típica (DT) para el sobrepeso y +2 DT para la obesidad en menores, siendo equivalentes a $25 \mathrm{~kg} / \mathrm{m}^{2}$ y $30 \mathrm{~kg} / \mathrm{m}^{2}$, respectivamente, en la escala de adultos (Monasta, Lobstein, Cole, Vignerová, \& Cattaneo, 2011).

\section{Análisis Estadístico}

Para el análisis de los resultados, la muestra de menores 
fue dividida en tres grupos de edad: G1 de entre cinco y siete años (n=977), G2 de entre ocho y 10 años $(n=1093)$ y G3 de entre 11 y 14 años $(n=1729)$.

La muestra se ha detallado mediante el empleo de estadísticos descriptivos (frecuencias, porcentajes, media, desviación típica y $95 \%$ de intervalo de confianza). El test de proporción chi-cuadrado fue empleado para comprobar la igualdad de proporciones de los grupos en una base de datos grande. Cuando las observaciones no contenían ningún valor, estas no fueron tenidas en cuenta por el programa para el análisis descriptivo.

Tests $\mathrm{T}$ han sido utilizados para hallar la existencia de diferencias significativas entre sexos para cada una de las variables continuas contempladas, y una prueba ANOVA para determinar las diferencias entre los diferentes grupos de IMC.

Todos los análisis estadísticos se realizaron con Stata para Mac, versión 12 y la significación estadística se estableció en un valor de $p<.05$ para todas las pruebas estadísticas.

\section{Aspectos Éticos}

Este estudio ha sido realizado con datos públicos anonimizados y sin posibilidad de identificar a los encuestados. De acuerdo con la legislación española, no es necesaria la aprobación de ningún Comité Ético.

\section{Resultados}

La muestra de estudio incluyó los datos de 7598 encuestas, $50 \%$ adultos $(n=3799)$ y $50 \%$ menores $(n=3799)$. En los adultos $(\mathrm{n}=3799)$, la distribución fue de $42.6 \%$ hombres y $57.4 \%$ de mujeres y su edad media de $41 \pm 11$ años. Finalmente, en el grupo de menores $(n=3799)$, la distribución fue de $50.8 \%$ de hombres y $49.2 \%$ de mujeres y una edad media de $9.8 \pm 2.9$ años. En cuanto a su IMC, los adultos presentaban, en conjunto, sobrepeso; sin embargo, los menores presentaban normopeso (Tabla 1). En el análisis del IMC de los menores diferenciado grupos de edad, el G1 tenía infrapeso $\mathrm{y}$, a medida que avanzaba el grupo de edad, el IMC avanzaba pero, en ambos grupos, dentro del normopeso (Tabla 1). En el análisis diferenciado por sexos, en el grupo de adultos se detectaron diferencias significativas entre ambos sexos $(p<$ .0001) siendo, de media, el IMC de los adultos varones de 26.3 en las mujeres de $24.6 \mathrm{~kg} / \mathrm{m}^{2}$. En menores no se hallaron

Tabla 1

Estadística descriptiva de la muestra de estudio (M: media; DT: desviación típica; IC 95\%: intervalo de confianza al 95\%)

\begin{tabular}{|c|c|c|c|c|c|c|}
\hline \multirow[b]{3}{*}{ Variable } & & \multicolumn{5}{|c|}{ Total $(\mathrm{n}=7598)$} \\
\hline & & \multirow{2}{*}{\multicolumn{2}{|c|}{$\begin{array}{l}\text { Adultos ( } \mathrm{n}=3799) \\
\text { M S DT (IC 95\%) }\end{array}$}} & \multicolumn{3}{|c|}{ Menores $(\mathrm{n}=3799)$} \\
\hline & $\mathbf{n}$ & & & $\mathbf{n}$ & \multirow{2}{*}{\multicolumn{2}{|c|}{ M }} \\
\hline$\overline{\text { Edad }}$ & 3799 & \multicolumn{2}{|c|}{$4111(40.6-41.4)$} & 3799 & & $9.82 .9(9.8-9.9)$ \\
\hline Peso & 3733 & \multicolumn{2}{|c|}{$71.915 .3(71.4-72.4)$} & 3569 & \multicolumn{2}{|c|}{$38.5 \quad 14.4(38-38.9)$} \\
\hline Altura & 3761 & \multicolumn{2}{|c|}{$168.29 .2(167.9-168.5)$} & 3499 & \multicolumn{2}{|c|}{$14219.5(141-143)$} \\
\hline IMC & 3713 & \multicolumn{2}{|c|}{$25.3-4.5(25.2-25.4)$} & 3397 & 18.84 & $(18.7-18.9)$ \\
\hline \multicolumn{7}{|c|}{ Menores $(\mathrm{n}=3,799)$} \\
\hline & \multicolumn{2}{|c|}{ G1 $(\mathrm{n}=977)$} & \multicolumn{2}{|c|}{ G2 $(n=1093)$} & \multicolumn{2}{|c|}{ G3 $(n=1729)$} \\
\hline Variable & n & $\begin{array}{c}\text { MSDT } \\
\text { (IC 95\%) }\end{array}$ & $\mathbf{n}$ & $\begin{array}{l}M \approx D T \\
\text { (IC 95\%) }\end{array}$ & n & $\begin{array}{l}\text { M } 1 \text { DT } \\
\text { (IC 95\%) }\end{array}$ \\
\hline Edad & 977 & $\begin{array}{l}60.8 \\
(6-6.1)\end{array}$ & 1093 & $\begin{array}{r}90.8 \\
(8.9-9.1)\end{array}$ & 1729 & $\begin{array}{c}12.51 .1 \\
(12.5-12.6)\end{array}$ \\
\hline Peso & 924 & $\begin{array}{l}23.8 \div 5.7 \\
(23.4-24.2)\end{array}$ & 1035 & $\begin{array}{c}34.688 .7 \\
(34.1-35.1)\end{array}$ & 1610 & $\begin{array}{c}49.411 .7 \\
(48.8-50)\end{array}$ \\
\hline Altura & 849 & $\begin{array}{c}118.1111 .5 \\
(117-119)\end{array}$ & 997 & $\begin{array}{l}13711.1 \\
(136-138)\end{array}$ & 1653 & $\begin{array}{c}157.3-11.1 \\
(157-158)\end{array}$ \\
\hline IMC & 837 & $\begin{array}{c}17.34 .96 \\
(17-17.6)^{a^{*} b^{*}}\end{array}$ & 976 & $\begin{array}{c}18.43 .7 \\
(18.1-18.6)^{\mathrm{a}^{*} \mathrm{c}^{8}}\end{array}$ & 1584 & $\begin{array}{c}19.93 .5 \\
(19.7-20.1)^{b^{*} c^{*}}\end{array}$ \\
\hline
\end{tabular}

G1: entre 5 y 7 años; G2: entre 8 y 10 años; G3: entre 11 y 14 años.

Edad (años). Peso (kg). Altura (cm). IMC: Índice de masa corporal $\left(\mathrm{kg} / \mathrm{m}^{2}\right)$.

a: G1 vs. G2; b: G1 vs. G3; c: G2 vs. G3.

$* p<.05$ diferencias significativas en el IMC entre ambos sexos $(p=$ .4).

En cuanto al nivel de estudios de los adultos encuestados: 472 (12.4\%) había finalizado los estudios primarios y/o la Educación Secundaria Obligatoria; 2431 (64\%) había superado el Bachillerato y/o algún Ciclo Formativo; $y$, finalmente, $896(23.6 \%)$ había realizado estudios universitarios.

\section{Relación entre el IMC del menor y nivel de estudios del adulto}

En la Figura 1 se puede observar cómo la proporción de menores con sobrepeso y obesidad desciende a medida que el nivel de estudios del adulto es más alto. Asimismo, la cantidad de menores con un valor de IMC relativo a infrapeso se incrementa ligeramente con el aumento de nivel de estudios del adulto.

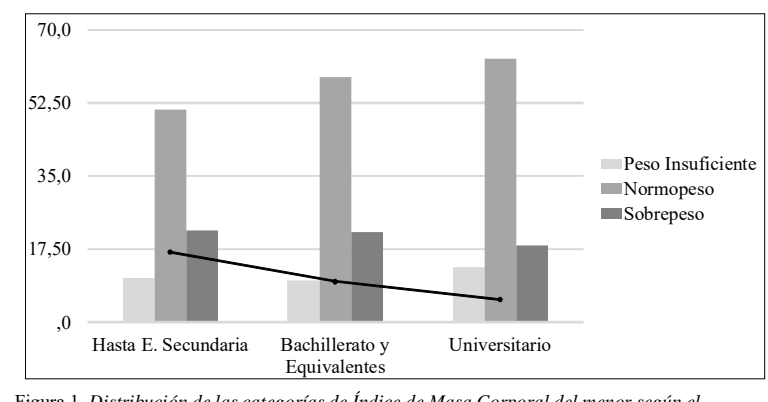

Figura 1. Distribución de las categorías de Índice de Masa Corporal del menor según el nivel de estudios del adulto

\section{Relación entre el IMC y frecuencia de AF de adultos y} menores

La Tabla 2 caracteriza a la muestra de adultos y a la de menores en relación a su IMC y la frecuencia con la que realizan algún tipo de AF. En general, los menores realizan con más frecuencia $\mathrm{AF}$ que los adultos $(38.1 \%$ de adultos sedentarios frente a $13.7 \%$ de menores sedentarios). Por grupos de edad, el G2 es el que presenta menor número de participantes sedentarios (11.2\%) y es el que realiza con mayor frecuencia $\mathrm{AF}$ (el $35.9 \%$ realiza $\mathrm{AF}$ varias veces a la semana). En siguiente lugar se encuentra el G3 y, finalmente, el G1 que, presenta un 2.5 puntos menos de incidencia del

\begin{tabular}{|c|c|c|c|c|c|c|c|c|c|c|c|}
\hline \multirow[b]{3}{*}{$\begin{array}{c}\text { IMC } \\
\text { Adulto } \\
\end{array}$} & \multicolumn{4}{|c|}{ Total $(\mathrm{n}=7598)$} & \multicolumn{7}{|c|}{ Menores $(\mathrm{n}=3799$ ) } \\
\hline & \multicolumn{2}{|c|}{$\begin{array}{c}\text { Adultos } \\
(\mathrm{n}=3799)\end{array}$} & \multicolumn{2}{|c|}{$\begin{array}{l}\text { Menores } \\
(\mathrm{n}=3799)\end{array}$} & \multicolumn{2}{|c|}{$\begin{array}{c}\text { G1 } \\
(\mathrm{n}=977)\end{array}$} & \multicolumn{2}{|c|}{$\begin{array}{c}\text { G2 } \\
(\mathrm{n}=1093)\end{array}$} & \multicolumn{2}{|c|}{$\begin{array}{c}\mathbf{G} 3 \\
(\mathrm{n}=1729)\end{array}$} & \multirow{2}{*}{$\begin{array}{c}\text { Total } \\
\begin{array}{c}\text { n } \\
(\%)\end{array}\end{array}$} \\
\hline & $\begin{array}{l}\mathrm{n} \\
(\%)\end{array}$ & M & $\begin{array}{c}\text { n } \\
(\%)\end{array}$ & M & $\begin{array}{c}n \\
(\%)\end{array}$ & M & $\begin{array}{c}\text { n } \\
(\%)\end{array}$ & M & $\begin{array}{c}\text { n } \\
(\%)\end{array}$ & M & \\
\hline IP & $\begin{array}{c}86 \\
(2.3)\end{array}$ & 17.3 & $\begin{array}{c}369 \\
(10.9)\end{array}$ & 13.7 & $\begin{array}{c}127 \\
(34.4)\end{array}$ & 12.8 & $\begin{array}{c}95 \\
(25.7)\end{array}$ & 13.1 & $\begin{array}{c}147 \\
(39.8)\end{array}$ & 14.8 & $\begin{array}{c}369 \\
(100)\end{array}$ \\
\hline $\mathrm{NP}$ & $\begin{array}{c}1850 \\
(49.8)\end{array}$ & 22.2 & $\begin{array}{c}1999 \\
(58.8)\end{array}$ & 17.6 & $\begin{array}{c}418 \\
(20.9)\end{array}$ & 15.7 & $\begin{array}{c}541 \\
(27.1)\end{array}$ & 16.8 & $\begin{array}{l}1040 \\
(52)\end{array}$ & 18.9 & $\begin{array}{l}1999 \\
(100)\end{array}$ \\
\hline S & $\begin{array}{c}1230 \\
(33.1)\end{array}$ & 27.1 & $\begin{array}{c}707 \\
(20.8)\end{array}$ & 21.6 & $\begin{array}{l}145 \\
(20.5)\end{array}$ & 18.6 & $\begin{array}{c}232 \\
(32.8)\end{array}$ & 20.8 & $\begin{array}{c}330 \\
(46.7)\end{array}$ & 23.4 & $\begin{array}{c}707 \\
(100)\end{array}$ \\
\hline $\mathrm{O}$ & $\begin{array}{c}547 \\
(14.7)\end{array}$ & 33.3 & $\begin{array}{l}322 \\
(9.5) \\
\end{array}$ & 25.9 & $\begin{array}{c}147 \\
(45.7)\end{array}$ & 24.6 & $\begin{array}{c}108 \\
(33.5)\end{array}$ & 25.6 & $\begin{array}{c}67 \\
(20.8)\end{array}$ & 29.3 & $\begin{array}{c}322 \\
(100)\end{array}$ \\
\hline $\begin{array}{c}\text { AF } \\
\text { Adulto }\end{array}$ & $\begin{array}{l}\mathrm{n} \\
(\%)\end{array}$ & & $\begin{array}{c}n \\
(\%)\end{array}$ & & $\begin{array}{c}\mathrm{n} \\
(\%)\end{array}$ & & $\begin{array}{c}\mathrm{n} \\
(\%)\end{array}$ & & $\begin{array}{c}\mathrm{n} \\
(\%)\end{array}$ & & \\
\hline $\mathrm{N}$ & $\begin{array}{c}1447 \\
(38.1)\end{array}$ & & $\begin{array}{c}541 \\
(13.7)\end{array}$ & & $\begin{array}{c}136 \\
(13.9)\end{array}$ & & $\begin{array}{c}122 \\
(11.2)\end{array}$ & & $\begin{array}{c}283 \\
(16.4)\end{array}$ & & \\
\hline $\mathrm{O}$ & $\begin{array}{c}1205 \\
(31.7)\end{array}$ & & $\begin{array}{c}806 \\
(17.6)\end{array}$ & & $\begin{array}{c}251 \\
(25.7)\end{array}$ & & $\begin{array}{c}226 \\
(20.7)\end{array}$ & & $\begin{array}{l}329 \\
(19)\end{array}$ & & \\
\hline $\mathrm{M}$ & $\begin{array}{c}556 \\
(14.6)\end{array}$ & & $\begin{array}{c}1157 \\
(21.6)\end{array}$ & & $\begin{array}{l}303 \\
(31)\end{array}$ & & $\begin{array}{c}352 \\
(32.2)\end{array}$ & & $\begin{array}{l}502 \\
(29)\end{array}$ & & \\
\hline $\mathrm{S}$ & $\begin{array}{c}591 \\
(15.6)\end{array}$ & & $\begin{array}{r}1294 \\
(25.9)\end{array}$ & & $\begin{array}{c}287 \\
(29.4)\end{array}$ & & $\begin{array}{c}392 \\
(35.9)\end{array}$ & & $\begin{array}{c}615 \\
(35.6)\end{array}$ & & \\
\hline Total & $\begin{array}{l}3799 \\
(100)\end{array}$ & & $\begin{array}{l}3397 \\
(100)\end{array}$ & & $\begin{array}{c}977 \\
(100)\end{array}$ & & $\begin{array}{l}1092 \\
(100)\end{array}$ & & $\begin{array}{l}1729 \\
(100)\end{array}$ & & \\
\hline
\end{tabular}


menor IMC del menor y mayor nivel estudios del adulto no había sido detectada previamente. Esta relación puede explicarse a que a mayor nivel educativo del adulto, más probable es que éste sea conocedor y más sensible a las recomendaciones sobre la práctica de AF y ser más consciente de su impacto para la salud presente y futura de su hijo y, de esta forma, inculca y promueve proactivamente este hábito en los menores bajo su tutela. Sin embargo, sí está comprobado que los adultos con menores a su cargo realizan menos AF que adultos sin hijos o con hijos mayores de edad (Adamo et al., 2012). Esto último, unido a la importancia que tiene en el niño el modelo de conducta de los padres (Clegg \& Legare, 2017) convierte, una vez más, a los adultos en la población diana sobre la que incidir políticas sanitarias de prevención primaria y secundaria para mejorar su salud y la de los niños que los rodean (y de los que aprenden modelos conductuales de auto-cuidado) (Ruiz-Ariza, de la Torre-Cruz, Suárez-Manzano, \& Martínez-López, 2019).

Paralelamente, también se asoció un menor IMC y mayor nivel de estudios del adulto con mayor frecuencia de AF en el menor otro hallazgo que concuerda con la explicación anterior de cómo a mayor nivel educativo del adulto, más probable es que éste tenga más conocimientos sobre estilo de vida saludable (sea más consciente del impacto en su calidad de vida) y, en consecuencia, los aplica a sí mismo (de ahí que tenga menor IMC) y a los menores con los que convive (de ahí que tengan hábitos de AF más saludables). Todo ello en concordancia con un estudio previo que identificó que la edad y la percepción de un estilo materno y paterno transformacional son predictores de la felicidad, bienestar y angustia psicológica en las chicas adolescentes (Ruiz-Ariza, de la Torre-Cruz, Appelqvist-Schmidlechner, Tamminen, \& Martínez-López, 2020).

En cuanto al análisis diferenciado por sexos, las mujeres ya desde la niñez muestran hábitos de AF más sedentarios que el de los hombres. Este fenómeno, ya identificado previamente en otras poblaciones (Badr, Lakha, \& Pennefather, 2017; García-Jaén, Sellés-Pérez, Cortell-Tormo, Férriz-Valero, \& Cejuela, 2019; Hall-López, 2020; Post \& Palacios, 2019), es un reflejo las diferencias inherentes en el rol de género que se inculca ya desde la infancia y se refleja, tanto en los métodos de enseñanza de la educación física en el entorno escolar y la oferta de AF en el tiempo libre diferenciada para niños y niñas, en la primera etapa vital; y para hombres y mujeres, en la etapa adulta (Fraguela-Vale, Varela-Garrote, \& VarelaCrespo, 2020; Ortega et al., 2018; Pinel et al., 2017).

\section{Fortalezas y limitaciones del estudio}

Este estudio tiene importantes limitaciones que deben ser reconocidas. Primero, se ha empleado información autoreportada sobre el peso, talla e IMC en lugar de mediciones realizadas por un evaluador experto. En segundo lugar, la población exclusivamente española limita la generalización de nuestros resultados a otras poblaciones. En tercer lugar, él hábito de AF también fue autorreportado y calificado por el participante en términos de frecuencia sin haberse cuantificado objetivamente mediante podómetro o acelerómetro en términos de cantidad, frecuencia e intensidad de la AF realizada.

A pesar de las limitaciones antes mencionadas, también hay fortalezas importantes. Se trata de un estudio poblacional amplio que, aunque con cautela, es representativa de la población española y reflejo real la situación actual de los hábitos de AF de las nuevas generaciones. Al mismo tiempo, es la primera vez que se detectan relaciones directas entre los hábitos de $\mathrm{AF}$ de los adultos y los menores con los que conviven en la población española, habiendo variables y asociaciones que se comportan de manera similar a la de otras poblaciones pero, en otros casos, habiendo particularidades para la población española.

\section{Conclusiones}

Independientemente, de que la obesidad sea una enfermedad multifactorial y que los factores socio-económicos sean determinantes en el estilo de vida de los niños y adolescentes; los resultados obtenidos sugieren que los hábitos de salud que mantienen los adultos con los que éstos conviven representan modelos de imitación para los menores.

Por tanto, las políticas e intervenciones de Salud Pública, deben tener como población diana a los menores para inculcar en ellos hábitos de AF saludables. Sin embargo, si se quiere que dichas políticas sean realmente eficaces deben incluir e integrar a los adultos, para infundir en los menores modelos de conducta saludables.

\section{Referencias}

Adamo, K. B., Langlois, K. A., Brett, K. E., \& Colley, R. C. (2012). Young children and parental physical activity levels: Findings from the Canadian health measures survey. American Journal of Preventive Medicine, 43(2), 168-175. doi: 10.1016/j.amepre.2012.02.032

Álvarez, O., Castillo, I., Molina-García, V. \& Balagué, G. (2016). Transformational leadership on the athletic field: An international review. Revista de Psicología del Deporte, 25(2), 319-326.

Badr, H. E., Lakha, S. F., \& Pennefather, P. (2017). Differences in physical activity, eating habits and risk of obesity among Kuwaiti adolescent boys and girls: A population-based study. International Journal of Adolescent Medicine and Health, 31(1): 20160138. doi: 10.1515/ijamh-2016-0138

Blundell, J., Dulloo, A., Salvador, J., \& Fruhbeck, G. (2014). Beyond BMI - phenotyping the Obesities. Obesity Facts, 7, 322-328. doi: $10.1159 / 000368783$

Cawley, J. (2015). An economy of scales: A selective review of obesity's economic causes, consequences, and solutions. Journal of Health Economics, 43, 244-268. doi: 10.1016/j.jhealeco.2015.03.001

Clegg, J. M., \& Legare, C. H. (2017). Parents scaffold flexible imitation during early childhood. Journal of Experimental Child Psychology, 153, 1-14. doi: 10.1016/j.jecp.2016.08.004

Cole, T. J., Flegal, K. M., Nicholls, D., \& Jackson, A. A. (2007). Body mass index cut offs to define thinness in children and adolescents: International survey. British Medical Journal (Clinical Research Edition), 335(7612), 194-202. doi: 10.1136/bmj.39238.399444.55

De Cocker, K., Ottevaere, C., Sjöström, M., Moreno, L. A., Wärnberg, J., Valtuena, J., ... Artero, E. G (2011). Self-reported physical activity in European adolescents: Results from the HELENA (Healthy Lifestyle in Europe by Nutrition in Adolescence) Study. Public Health Nutrition, 14(2), 246-254. doi: 10.1017/S1368980010000558.

De Onis, M., Blössner, M., \& Borghi, E. (2010). Global prevalence and trends of overweight and obesity among preschool children. The American Journal of Clinical Nutrition, 92(5), 1257-1264. doi: 10.3945/ajcn.2010.29786

Erickson, K. I., Voss, M. W., Prakash, R. S., Basak, C., Szabo,A., Chaddock, L., ... Kramer, A. F. (2011). Exercise training increases size of hippocampus and improves memory. Proceedings of the National Academy of Sciences of the United States of America, 108(7), 3017- 
3022. doi: $10.1073 /$ pnas. 1015950108

Finegood, D. T., Merth, T. D., \& Rutter, H. (2010). Implications of the foresight obesity system map for solutions to childhood obesity. Obesity, 18(S1), S13-S16. doi: 10.1038/oby.2009.426

Fradejas, E., \& Espada, M. (2018). Evaluation of motivation in teenagers who practice sports in school age. Retos: nuevas tendencias en educación física, deporte y recreación, 33, 27-33.

Fraguela-Vale, R., Varela-Garrote, L. \& Varela-Crespo, L. (2020). Perfiles de ocio deportivo en jóvenes españoles (15-20 años): un análisis de género. Retos: nuevas tendencias en educación física, deporte y recreación, 37, 419-426.

García-Jaén, M., Sellés-Pérez, S., Cortell-Tormo, J. M., Férriz-Valero, A., \& Cejuela, R. (2019). Evaluación de los patrones de movimiento fundamentales en niños: comparación de género en escolares de Educación Primaria. Retos: nuevas tendencias en educación física, deporte y recreación, 34, 282-286.

Garrido-Miguel, M., Cavero-Redondo, I., Álvarez-Bueno, C., RodríguezArtalejo, F., Moreno, L. A., Ruiz, J. R., ... Martínez-Vizcaíno, V. (2019). Prevalence and trends of overweight and obesity in European children from 1999 to 2016: A systematic review and meta-analysis. JAMA Pediatrics, 173(10), e192430-e192430. doi: 10.1001/ jamapediatrics.2019.2430

Gearin, B. M., \& Fien, H. (2016). Translating the neuroscience of physical activity to education. Trends in Neuroscience and Education, 5(1), 12-19. doi: $10.1016 /$ j.tine.2016.02.001

Godoy-Cumillaf, A., Bruneau-Chávez, J., Fuentes-Merino, P., \& OjedaSantana, D. (2020). Association between body mass index of parents and children. Retos: nuevas tendencias en educación física, deporte y recreación, 38, 708-712.

Gómez Pinilla, F., \& Hillman, C. (2013). The influence of exercise on cognitive abilities. Comprehensive Physiology, 3(1), 403-428. doi: 10.1002/cphy.c110063

Hillman, C. H., Pontifex, M. B., Castelli, D. M., Khan, N. A., Raine, L. B., Scudder, M. R., ... Kamijo, K. (2014). Effects of the FITKids randomized controlled trial on executive control and brain function. Pediatrics, 134(4), e1063-e1071. doi: 10.1542/peds.2013-3219

Hall-López, J. A. (2020). Secondary physical education, participation by sex in moderate to vigorous physical activity. Retos: nuevas tendencias en educación física, deporte y recreación, 38, 543-546.

Instituto Nacional de Estadística. (2020). Encuesta Nacional de Salud de España 2017. Madrid: Instituto Nacional de Estadística. https:// www.mscbs.gob.es/estadEstudios/estadisticas/encuestaNacional/ encuesta2017.htm1

Kaseva, K., Hintsa, T., Lipsanen, J., Pulkki-Råback, L., Hintsanen, M., Yang, X., ... Keltikangas-Järvinen, L. (2017). Parental physical activity associates with offspring's physical activity until middle age: A 30year study. Journal of Physical Activity and Health, 14(7), 520-531. doi: 10.1123/jpah.2016-0466

Lane, M., Zander-Fox, D. L., Robker, R. L., \& McPherson, N. O. (2015). Peri-conception parental obesity, reproductive health, and transgenerational impacts. Trends in Endocrinology \& Metabolism, 26(2), 84-90. doi: 10.1016/j.tem.2014.11.005

Lee, C. Y., Ledoux, T. A., Johnston, C. A., Ayala, G. X., \& O'Connor, D. P. (2019). Association of parental Body Mass Index (BMI) with child's health behaviors and child's BMI depend on child's age. BMC Obesity, 6(1), 11-21. doi: 10.1186/s40608-019-0232-x

Manios, Y., Androutsos, O., Katsarou, C., Vampouli, E. A., Kulaga, Z., Gurzkowska, B., ... Koletzko, B. (2018). Prevalence and sociodemographic correlates of overweight and obesity in a large Pan-European cohort of preschool children and their families: The ToyBox study. Nutrition, 55, 192-198. doi: 10.1016/j.nut.2018.05.007

Martínez-Baena, A. C. M., Mayorga-Vega, D. M., \& Viciana, J. (2018). Factores predictores de la actividad física en escolares españoles de acuerdo a su estado de peso. Retos: nuevas tendencias en educación física, deporte y recreación, 33, 74-80.

Martínez-López, E. J., Moreno-Cerceda, J., Suárez-Manzano, S., \& RuizAriza, A. (2018). Efecto y satisfacción de un programa de actividad física controlada por pulsómetro en el índice de masa corporal de escolares con sobrepeso-obesidad. Retos: nuevas tendencias en educación física, deporte y recreación, 33, 179-184.

Monasta, L., Lobstein, T., Cole, T. J., Vignerová, J., \& Cattaneo, A.
(2011). Defining overweight and obesity in pre school children: IOTF reference or WHO standard? Obesity Reviews, 12(4), 295300. doi: 10.1111/j.1467-789X.2010.00748.x

Morton, K. L., Wilson, A. H., Perlmutter, L. S. \& Beauchamp, M. R. (2012). Family leadership styles and adolescent dietary and physical activity behaviors: A cross-sectional study. International Journal of Behavioral Nutrition and Physical Activity, 9, 48-56. doi: 10.1186/ 1479-5868-9-48

NCD Risk Factor Collaboration. (2017). Worldwide trends in children's and adolescents' body mass index, underweight, overweight and obesity, in comparison with adults, from 1975 to 2016: A pooled analysis of 2,416 population-based measurement studies with 128.9 million participants. Lancet, 390, 2627-2642. doi: 10.1016/S01406736(17)32129-3

Oliver, M., Schofield, G M., \& Kolt, G S. (2007). Physical activity in preschoolers: Understanding prevalence and measurement issues. Sports Medicine, 37(12), 1045-1070. doi: 10.2165/00007256200737120-00004

Ortega, F. Z., Jiménez, J. L. U., Molero, P. P., Valero, G. G., Sánchez, M. C., \& Cuberos, R. C. (2018). Niveles de actividad física en alumnado de Educación Primaria de la provincia de Granada. Retos: nuevas tendencias en educación física, deporte y recreación, 34, 218-221.

Pinel, M., Chacón, R., Castro, M., Espejo, T., Zurita, F., \& Pérez, A. (2017). Diferencias de género en relación con el Índice de Masa Corporal, calidad de la dieta y actividades sedentarias en niños de 10 a 12 años. Retos: nuevas tendencias en educación física, deporte y recreación, 31, 176-180.

Popper, M. \& Mayseless, O. (2003). Back to basics: Applying parenting perspective to transformational leadership. Leadership Quarterly, 14(1), 41-65. doi:10.1016/S1048-9843(02)00183-2

Post, P., \& Palacios, R. (2019). Aggie play: A gender-relevant physical activity program for girls. Journal of Sport and Exercise Psychology, 41(4), 194-205. doi: 10.1123/jsep.2018-0299

Reilly, J. J., \& Kelly, J. (2011). Long-term impact of overweight and obesity in childhood and adolescence on morbidity and premature mortality in adulthood: Systematic review. International Journal of Obesity, 35(7), 891-898. doi: 10.1038/ijo.2010.222

Rosa, A., García, E., \& Martínez, H. (2020). Influencia de un programa de actividad física sobre la atención selectiva y la eficacia atencional en escolares. Retos: nuevas tendencias en educación física, deporte y recreación, 38, 560-566.

Ruiz-Ariza, A., de la Torre-Cruz, M. J., Appelqvist-Schmidlechner, K., Tamminen, N., \& Martínez-López, E. J. (2020). Does physical fitness and parenting transformational style predict happiness and wellbeing and distress in adolescents? Retos: nuevas tendencias en educación física, deporte y recreación, 38, 626-631.

Ruiz-Ariza, A., de la Torre-Cruz, M., Suárez-Manzano, S., \& MartínezLópez, E. J. (2019). Apoyo hacia la actividad física y rendimiento académico independientemente del estatus socioeducativo parental. Retos: nuevas tendencias en educación física, deporte y recreación, 38, 208-212.

Schoeppe, S., Röbl, M., Liersch, S., Krauth, C., \& Walter, U. (2016). Mothers and fathers both matter: The positive influence of parental physical activity modeling on children's leisure-time physical activity. Pediatric Exercise Science, 28(3), 466-472. doi: 10.1123/pes.20150236

Verma, N., Eklund, R. C., Arthur, C. A., Howle, T. C. \& Gibson, A. (2019). Transformational teaching, self-presentation motives, and identity in adolescent female physical education. Journal of Sport and Exercise Psychology, 41(1), 1-9 doi:10.1123/jsep.2017-0299

Wang, Y., \& Lim, H. (2012). The global childhood obesity epidemic and the association between socio-economic status and childhood obesity. International Review of Psychiatry, 24(3), 176-188. doi: 10.3109/ 09540261.2012.688195

Warkentin, S., Mais, L. A., Latorre, M. R. D., Carnell, S., \& Taddei, J. A. A. C. (2018). Parents matter: Associations of parental BMI and feeding behaviors with child BMI in Brazilian preschool and schoolaged children. Frontiers in Nutrition, 5, 69. doi: 10.3389/ fnut.2018.00069

World Health Organization. (2019). Global action plan on physical activity 2018-2030: More active people for a healthier world, Geneva, Switzerland: World Health Organization. 